\title{
Prescribing Psychologists in Integrated Primary Care: Introduction to the Special Section
}

\author{
Steven R. Tulkin
}

Published online: 29 November 2012

(c) Springer Science+Business Media New York 2012

\begin{abstract}
The authority to prescribe could differentiate psychologists from other non-physicians in primary care, achieving a significant step for the growth of our profession. Psychologists are currently prescribing in primary care settings in a number of public service venues, as well as medical school training facilities. In this Special Section of the Journal of Clinical Psychology in Medical Settings, results of a survey of physicians working with a prescribing psychologist indicate that the psychologist is highly valued and seen as a competent prescriber. A series of papers providing case examples demonstrate how psychologists are evolving in these new clinical roles, as well as participating in behavioral and psychopharmacology training of primary care physicians.
\end{abstract}

Keywords Prescribing psychologist - Integrated care · Primary care $\cdot$ Behavioral health

\section{Introduction}

McGrath and Sammons (2011) discuss the importance of psychologists acquiring the skills to practice in primary care settings. They note that "Primary care psychology is distinct from the mental health and health psychology models in that... the primary care psychologist is a full participant in the primary medical care, providing varying interventions for patients with various types of problems including acute medical conditions" (p. 115). In order to be

S. R. Tulkin $(\bowtie)$

California School of Professional Psychology at Alliant International University, One Beach Street, San Francisco, CA 94133-1221, USA

e-mail: stulkin@alliant.edu hired into the new positions being created for behavioral consultants in primary care, psychologists must offer a skill set that differentiates them from other healthcare professionals who have been trained to work in primary care (e.g., nurses and masters level mental health providers). McGrath and Sammons list nine functions of the primary care psychologist "which combine the traditional skills of the psychologist with new skills relevant to the primary care setting." However, seven of the nine clinical skills are also included in the curricula for programs in nursing and professional masters level counseling (although likely with less breadth and depth of skills than would be found for psychologists). The two remaining functions, relating to outcomes assessments and research protocols, are unique to psychologists, but are unlikely to be the top priority in primary care clinical settings.

One area where primary care physicians (PCPs) indicate they need help is in differential diagnosis and treatment of patients with mental health disorders, and in the management of patients with co-morbid medical and mental health conditions. More than $60 \%$ of family medicine residencies offer no formal training in psychopharmacology (Bazaldua et al., 2005), yet primary care is the place where most prescriptions for psychoactive medications are written. One study (Mark, Levit, \& Buck, 2009) found that approximately $60 \%$ of prescriptions for psychotropic medications are written by PCPs. The number of psychologists who are licensed to prescribe is growing, in the two states that have approved legislation, and in Federal jurisdictions (Department of Defense, Public Health Service, and Indian Health Service). Recent estimates indicate that there are close to 100 psychologists authorized to prescribe (McGrath, 2010). Most prescribing psychologists are in mental health settings, including private practice. However, a number of public service agencies have welcomed prescribing 
psychologists into primary care. McGrath and Sammons (2011) note that "the combination of prescriptive authority, an understanding of psychosocial diagnosis and intervention, and behavioral management skills will enhance the attractiveness of psychologists as partners to PCPs" (p. 118). They suggest further that prescribing psychologists in primary care will enhance psychology's "contribution to the public good through enhancement of services for individuals with emotional and behavioral concomitants to their physical disorders" (p. 118).

DeLeon (2012) predicts that "we will continue to see an increasing number of non-physician primary care providers adopting the doctoral level of training as their standard, and expanding their scopes of practice to fully utilize their clinical expertise" (p. 5). He goes on to say that "the right to prescribe medications by nurse practitioners and doctors of nursing practice, as well as by clinical pharmacists, continues to mature exponentially across the nation" (p. 5). These professionals are also being trained in behavioral interventions, and the implication is that for psychologists to be competitive for positions in primary care, prescriptive authority is an advantage.

Psychologists have recently joined the ranks of professionals authorized to prescribe in primary care. The purpose of this Special Section of the Journal of Clinical Psychology in Medical Settings (JCPMS) is to describe the first steps in this important process, reporting the results of a survey of primary care providers asking for their evaluation of the services of a prescribing psychologist and then describing the integration of primary care prescribing psychologists into primary care in several public service settings. The first paper, by Shearer, Harmon, Seavey and Tiu, describes the Primary Care Prescribing Psychologist (PCPP) model in which a prescribing psychologist is embedded in a primary care clinic. This paper discusses the strengths and weaknesses of the model, as reported by medical providers on the primary care team. Each of the next four papers describes the work of the prescribing psychologist in various settings: Shearer at Madigan Hospital, the primary medical center of Army Medical Command's Western Region; McGuinness at the Chaparral Medical Center, one of seven medical centers in The La Clinica de Familia, a federally qualified health center in a rural US-Mexico border town; and Sutherland and Tulkin at the Indian Health Service Crow/N. Cheyenne Hospital, Crow Agency Montana. The final paper, by Hoover and Andazola describes the collaborative roles of a prescribing psychologist (Hoover) and Family Practice Physician (Andazola) in the training of family medicine residents and prescribing psychologists.

Following these papers is a commentary from Robert McGrath, Ph.D. who is an acknowledged leader in both the prescription authority movement for psychologists and the efforts for integrated health care. Dr. McGrath has written extensively about professional issues in psychology.

\section{References}

Bazaldua, O., Ables, A. Z., Dickerson, L. M., Hansen, L., Harris, I., Hoehns, J., et al. (2005). Suggested guidelines for pharmacotherapy curricula in family medicine residency training. Recommendations from the Society of Teachers of Family Medicine Group on Pharmacotherapy. Family Medicine, 37, 99-104.

DeLeon, P. (2012). An impressive evolution. National Psychologist, $21,5$.

Mark, T. L., Levit, K. R., \& Buck, J. A. (2009). Psychotropic drug prescriptions by medical specialty. Psychiatric Services, 60, 1187.

McGrath, R. E. (2010). Prescriptive authority for psychologists. Annual Review of Clinical Psychology, 6, 21-47.

McGrath, R., \& Sammons, M. (2011). Prescribing and primary care psychology: Complementary paths for professional psychology. Professional Psychology, 42, 113-120. 